\title{
Are We At The End of the Antimicrobial Era?
}

\author{
Samuel N. Osei-Djarbeng \\ Dean, Faculty of Health Sciences, Kumasi Technical University, Ghana
}

Antimicrobials have helped prolong the life expectancy of man for many years. Until the discovery of these agents that are capable of inhibiting the growth of micro-organisms or killing them altogether, man suffered incessantly from infections of various aetiology.

Historically, several people died from infections caused by various species of bacteria, fungi, viruses, protozoa and other parasites. The discovery of antimicrobials, especially the antibiotics (antibacterial agents) revolutionised management and treatment of infectious diseases. Taking the antibiotics for example, the discovery of the penicillins by Alexander Fleming in 1928 brought relief to many who suffered from bacterial infections. It was not until 1945 that the penicillins were produced in commercial quantities to combat infections caused by Staphylococcus aureus and other bacteria. Though penicillin, as it were, was not the first antibacterial agent discovered, its serendipitous discovery from another microorganism (mould) gave hope that other classes of antimicrobials could be obtained from such sources. Indeed, research in finding more antimicrobials from other microbial (mainly fungal) sources intensified, and this led to discovery (originally) of many classes of antimicrobials including the cephalosporins, aminoglycosides, tetracyclines, chloramphenicol.

By the mid-1960s most of these classes of antibiotics had been discovered, and it became apparent that the morbidity and mortality associated with infectious diseases had greatly abated. This feat gave a general impression that by the turn of the then twentieth century (that is by beginning of the twenty first century), infectious diseases would be a thing of the past. Unfortunately, this never came to be. Seventeen years into the new century, and almost all the existing antimicrobial agents have encountered resistance by microorganism.

In an interview with The New York Times in 1945 after receiving the Nobel Prize, Alexander Fleming, who discovered penicillin, predicted that the inappropriate use of penicillin could result in resistant mutant forms of $S$. aureus that could be more serious in the host. It did not take long after this prediction before $S$. aureus acquired enzyme that made them refractory to penicillins. Today, it is becoming more and more difficult and/or expensive to manage infections that hitherto were easily treatable. Increasingly the medical profession is now faced with the problem of treating challenging infections, such as those caused by hospital- and community-acquired meticillin-resistant $S$. aureus (MRSA) or multidrug-resistant (MDR) Gram-negative bacteria, and this is a major concern.

\section{How Did We Get Here?}

The major underlying factor of resistance to antimicrobials is their misuse. Over the years, at least one mechanism of resistance has been reported for each of the classes of antibiotics known. The resistance of micro-organisms to antimicrobial agents can be categorised into three, namely; genotypic, in which micro-organism carries a certain genetic resistance elements; phenotypic where microorganisms can stay alive and survive above a certain amount of antimicrobial agents in the laboratory, and; clinical where microbes are able to proliferate in human (host) during therapy in the presence of antimicrobials.

Needless prescription of antimicrobials has contributed significantly to resistance. Studies have shown that about one in two of prescriptions written in consulting rooms are for sicknesses that do not require antibiotics. This includes prescription of antibiotics for viral infections, which the medication may not have any activity against; and antibiotics for some allergic conditions which may manifest as cough. In a study at the United States of America, it was found that $30-60 \%$ of antibiotics prescribed 
in intensive care units (ICUs) were unnecessary, inappropriate, or suboptimal ${ }^{1}$. In most health facilities in developing countries, because of poor diagnosis broad-spectrum antibiotics are prescribed. Indeed if proper diagnosis is established, a narrow-spectrum one that may target the causative organism only may be preferable.

Additionally, feeding livestock, poultry, fish and other animals with food containing antibiotics with an aim to build them up and protect them from diseases has also added to the menace of resistance. Further, patients have played a major role in antimicrobial resistance by failing to observe instructions on dosage and duration of therapy for antimicrobials. Most antibiotics are to be taken for a specific period of time at given intervals. In instances where a patient does not complete the full course of the therapy, and at the given intervals, the bacteria may survive and become resistant to that antibiotic. In some countries and communities, especially developing ones, there is indiscriminate access to antibiotics, and they are not properly used.

\section{How can we reverse the Trend?}

Many approaches can be used to reduce the incidence and spread of antimicrobial resistance. These include approaching the issue from the prescribers' perspective, the patients' perspective, public education and the need for newer antimicrobial agents with, perhaps, totally different mechanisms of action from the existing ones.

Rational prescription of antimicrobials is required. Prescribers must prescribe antimicrobials based on the micro-organism in question. That is to say that proper diagnosis must be done, and the antimicrobial must be given at the right dosage and the right duration. Hygienic practices such as hand washing, wearing of gloves, use of disinfectants, etc, must be followed. Immunizations can also be encouraged.

The patients' role in reducing the incidence of resistance lies on compliance with antimicrobial medication. Patients are encouraged to complete the regimen, even if they feel better before completion of treatment. Antimicrobials should not be requested from pharmacies without prescriptions (and pharmacists and pharmacy assistants should not give them out without prescriptions).

Additionally, there must be public education on the use of antimicrobials. For example people should be educated not to use antibiotics for treatment of common cold or allergy. People should not on their go and request for antibiotics from pharmacies, especially in countries where the rules governing dispensing of Prescription Only Medicine (POM) are slack.

Finally, research must be encouraged to search for newer antimicrobial agents, perhaps from higher plants, animals and animal products. The use of defensin to boost the individuals' ability to fight off infections, and anti-quorum sensing agents to disrupt the communication network among microbes can also be promoted to help manage infections to reduce resistance.

\section{Conclusion}

In conclusion, society would not want to turn back the clock to the era when there were no antimicrobial agents, when many people died from simple wounds as a result of infections, when many died from malaria, when infant mortality was high, when simple surgery was so dreaded. Society wishes to live in an era where antimicrobials would perfectly work again, when resistance may be no more. And this can be achieved by a collective effort of all so that society would not be at the end of the antimicrobial era.

\section{Reference}

[1] Luyt C.E., Brechot N., Trouillet J.L. and Chastre J. (2014). Antibiotic stewardship in the intensive care unit. Crit Care, 18(5):480. 